\title{
Reprodução de Meloidogyne spp. em porta-enxertos e híbridos de pepino
}

\author{
Silvia Renata S Wilcken'; Juliana MO Rosa'; ${ }^{1}$ Andréa RO Higuti'; Maria José de M Garcia ${ }^{2}$; Antonio \\ Ismael Inácio Cardoso ${ }^{1}$ \\ ${ }^{1}$ UNESP-FCA-Depto. Prod. Vegetal. C. Postal 23, 18610-307 Botucatu-SP; ${ }^{2}$ APTA-Pólo Centro Oeste, Unid. de Pesquisa e Desenvolvi- \\ mento de Bauru, Av Rodrigues Alves 40-40, 17030-000 Bauru-SP; srenata@fca.unesp.br
}

\section{RESUMO}

A enxertia é uma técnica alternativa frequentemente recomendada para a cultura do pepino em áreas infestadas com nematóides das galhas. O presente trabalho teve como objetivo determinar o fator de reprodução de Meloidogyne javanica e de Meloidogyne incognita raça 2 em seis porta-enxertos para pepino (abóbora 'Menina Brasileira', moranga 'Exposição', 'Shelper', 'Tetsukabuto', 'B8-A Tetsukabuto' e 'Excite Ikki') e quatro híbridos de pepino (Cucumis sativus) tipo japonês ('Yoshinari', 'Kouki', 'Taisho' e 'Tsuyataro'). Foram conduzidos dois experimentos em casa-de-vegetação, um com cada espécie do nematóide, sendo cada parcela constituída de uma planta mantida em vaso contendo 2 litros de solo autoclavado. Nove dias após transplante, cada planta foi inoculada com 5.000 ovos e juvenis de segundo estádio (população inicial - Pi) de $M$. javanica ou M. incognita raça 2. Tomateiros 'Rutgers' foram utilizados como padrão de viabilidade do inóculo, em ambos os experimentos. $\mathrm{O}$ delineamento experimental foi inteiramente casualizado com cinco repetições por tratamento. Sessenta dias após a inoculação, cada planta foi avaliada, quanto ao peso fresco da raiz, número total de nematóides presentes no solo e na raiz (população final - Pf), número de nematóides/g de raiz e fator de reprodução de ambas as espécies de Meloidogyne (FR=Pf/Pi). Todos os porta-enxertos e híbridos de pepino testados apresentaram fatores de reprodução superiores a um, proporcionando a multiplicação de $M$. javanica e de $M$. incognita raça 2 , porém, os valores nos híbridos de pepino foram superiores aos dos porta-enxertos.

Palavras-chave: Cucumis sativus, Meloidogyne javanica, Meloidogyne incognita, enxertia.

\section{ABSTRACT}

Reproduction of Meloidogyne spp. in rootstocks and cucumber hybrids

Grafting is an alternative technique often recommended for the cucumber crop in root-knot nematodes infested areas. This study aimed to determine the reproduction factor of Meloidogyne javanica and $M$. incognita race 2 on six rootstocks for cucumber (squash 'Menina Brasileira, pumpkim 'Exposição', 'Shelper', 'Tetsukabuto', 'B8-A Tetsukabuto' and 'Excite Ikki') and four cucumber (Cucumis sativus) Japanese type hybrids of ('Yoshinari', 'Kouki', 'Taisho' and 'Tsuyataro'). Two experiments were carried out in greenhouse, each one with a nematode specie. Each plot consisted of one plant per pot containing 2 liters of autoclaved soil. Nine days after the seedlings transplantation, each plant was inoculated with 5,000 eggs and second-stage juveniles (initial population - Pi) of M. javanica or M. incognita race 2. 'Rutgers' tomatoes were used as a standard for inoculum viability in both experiments. The experimental design was completely randomized with five replicates per treatment. Sixty days after inoculated, each plant was evaluated, when root fresh weight, total number of nematodes in the soil and in the roots (final population - Pf), nematodes number per gram of root and the reproduction factor of both Meloidogyne species $(\mathrm{FR}=\mathrm{Pf} / \mathrm{Pi})$ were determined. All rootstocks and cucumber hybrids allowed the $M$. javanica and $M$. incognita race 2 multiplication, but, generally, reproduction factor values were greater in cucumbers than in rootstocks.

Keywords: Cucumis sativus, Meloidogyne javanica, Meloidogyne incognita, grafting.

(Recebido para publicação em 12 de maio de 2009; aceito em 3 de fevereiro de 2010) (Received on May 12, 2009; accepted on February 3, 2010)

O pepino (Cucumis sativus L.) é uma espécie adaptada ao cultivo sob temperaturas superiores a $20^{\circ} \mathrm{C}$. Temperaturas inferiores afetam o desenvolvimento e a produtividade da cultura. Além disso, a necessidade de obtenção do produto no inverno, quando os preços são mais elevados, levou os produtores brasileiros, localizados em regiões sujeitas a baixas temperaturas, a cultivar pepino em ambiente protegido a partir da década de 80 (Cañizares, 1998; Cardoso \& Wilcken, 2008). Entre as cucurbitáceas, o pepino é a espécie mais cultivada sob essas condições em todo o mundo (Robinson \& Decker-Walters, 1999).

Embora o cultivo de pepino em ambientes protegidos contribua para o aumento da produtividade e qualidade do produto comercializado, quando feito de maneira intensiva, propicia o aumento de problemas relacionados à sanidade da cultura (Cañizares \& Goto, 1998). Dentre os patógenos do solo que afetam o pepino, os fitonematóides, são responsáveis por aproximadamente $67,7 \%$ dos replantios necessários em hortaliças (Oda, 1995).

Huang \& Viana (1980), estudando a suscetibilidade de pepino 'Aodai Melhorado’ a $M$. incognita em diferentes níveis populacionais, verificaram que um mês após a semeadura, $100 \%$ das plântulas inoculadas com 20.000 e 100.000 ovos/L morreram, e quando foram inoculadas com 1.000 ovos de $M$. incognita/L de solo houve redução superior a 50\% na produção de frutos de pepino.

Devido à falta de cultivares de pepinos comerciais com resistência a $M$. incognita e $M$. javanica, espécies mais freqüentes em cultivo protegido, outras medidas de controle têm sido recomendadas, dentre estas a desinfecção do solo com produtos químicos, rotações de cultura com espécies vegetais não hospedeiras de nematóides e técnicas culturais como a enxertia (Cardoso \& Wilcken, 2008; Cardoso, 2009).

A enxertia em pepinos é uma técnica que propicia, dependendo da combi- 
nação enxerto/porta-enxerto, melhoria da qualidade dos frutos com a retirada de cera, resultando em maior brilho, aumento do vigor da copa da planta e conseqüente aumento de produção, aumento da resistência do porta-enxerto a fatores adversos, menor incidência de doenças fúngicas do solo e infestação por nematóides (Kawaide, 1985; Cañizares \& Goto, 1998).

A técnica de enxertia também tem sido considerada uma alternativa freqüentemente recomendada para a cultura do pepino em áreas infestadas com nematóides das galhas. As taxas de multiplicação de $M$. javanica e $M$. incognita nos porta-enxertos recomendados ainda são desconhecidas, embora já tenham sido determinadas em diferentes cultivares de pepino plantadas em pé-francos em estufa e no campo, variando estas de 66,0 a 170,0 em estufa e de 3,0 a 59,7 a campo (Charchar et al., 2005).

O presente trabalho teve como objetivo determinar o fator de reprodução de Meloidogyne javanica e $M$. incognita raça 2 em seis porta-enxertos e quatro híbridos de pepino.

\section{MATERIAL E MÉTODOS}

Dois experimentos, $\operatorname{com} M$.javanica e $M$. incognita raça 2 , foram conduzidos separadamente em casa-de-vegetação da UNESP em Botucatu-SP.

Populações puras das espécies foram isoladas a partir da retirada da massa de ovos de cada fêmea devidamente identificada e multiplicadas em raízes de tomateiro 'Rutgers' em vasos com substratos previamente autoclavado, em casa-de-vegetação.

A população de $M$. javanica foi isolada a partir de raízes de pimentão 'Magali' proveniente do município de Santa Rosa-RS; e a população de $M$. incognita raça 2 a partir de raízes de cafeeiro provenientes do município de Osvaldo Cruz-SP.

Ovos e eventuais juvenis infectantes de $M$. javanica ou $M$. incognita raça 2 foram extraídos das raízes de tomateiro 60 dias após a inoculação, seguindo o método de extração proposto por Hussey \& Barker (1973), modificado por Bonetti \& Ferraz (1981), que consiste em pro- cessar o sistema radicular infectado em liquidificador com hipoclorito a 0,5\% e, em sequência, passar o processado por um jogo de peneiras de 20, 80 e 500 mesh, para a obtenção da suspensão de inóculo que é constituída pelos ovos e eventuais juvenis retidos na peneira de 500 mesh.

Foram avaliadas seis cultivares de abóboras e morangas recomendadas para utilização como porta-enxerto para pepino ('Menina Brasileira', 'Moranga Exposição', 'Shelper', 'Tetsukabuto', 'B8-A Tetsukabuto' e 'Excite Ikki') e quatro híbridos de pepino tipo japonês ('Yoshinari', 'Kouki', 'Taisho' e 'Tsuyataro').

Sementes destes dez tratamentos foram semeadas em bandeja de poliestireno expandido (isopor) de 128 células com substrato comercial (Plantmax ${ }^{\circledR}$ ). O transplante das mudas foi efetuado quando as mesmas apresentavam aproximadamente $5 \mathrm{~cm}$ de altura para vasos de 2 L contendo substrato composto por solo, areia e esterco bovino, na proporção $1: 2: 1$, previamente autoclavado.

A inoculação foi realizada nove dias após o transplante das mudas das cultivares de abóboras e/ou morangas e dos híbridos de pepino com 5.000 ovos e juvenis de segundo estádio do nematóide estudado/vaso (População inicial= Pi) suspensos em $2 \mathrm{~mL}$. A inoculação foi realizada em dois orifícios com 2 $\mathrm{cm}$ de profundidade, ao redor da muda. Tomateiros 'Rutgers' foram utilizados como padrão de viabilidade do inóculo em ambos os experimentos.

Os experimentos foram conduzidos em casa-de-vegetação, sendo cada parcela constituída de uma planta por vaso. $\mathrm{O}$ delineamento experimental foi inteiramente casualizado com cinco repetições por tratamento.

Sessenta dias após a inoculação, os sistemas radiculares de cada parcela foram separados da parte aérea, lavados e pesados após a retirada do excesso de umidade com auxílio de papel toalha. Em seguida, foram submetidos à solução de floxine B por 15 minutos, para a coloração das massas de ovos externa dos nematóides, as quais tiveram seus números contados juntamente com os números de galhas, ambos relacionados com a escala de notas proposta por
Taylor \& Sasser (1978). Por esta escala, são conferidas as notas $0,1,2,3,4$ e 5 se o número de galhas (ou massas de ovos) for nulo, 1-2, 3-10, 11-30, 31-100 e $>100$, respectivamente.

Em seguida, foram processados 250 $\mathrm{mL}$ de solo e todo o sistema radicular de cada parcela, de acordo com a metodologia flutuação, peneiramento e centrifugação e processamento em liquidificador, peneiramento e centrifugação, descritas por Jenkins (1964) e Coolen \& D'Herde (1972), respectivamente. O número total de nematóides presentes no solo e na raiz de cada parcela (População final= Pf) foi obtido através da multiplicação do número de nematóides presentes em $250 \mathrm{~mL}$ de solo por oito, para estimar em 2 L, e em seguida somado ao número de nematóides presentes na raiz. A partir destes dados calcularam-se os fatores de reprodução $(\mathrm{FR}=\mathrm{Pf} / \mathrm{Pi})$. As plantas que apresentaram valor de FR maior ou igual a 1,0, foram classificadas como plantas hospedeiras e, aquelas que apresentaram FR menor que 1,0, como plantas não hospedeiras (Oostenbrink, 1966).

Os dados obtidos foram submetidos à análise da variância e as médias comparadas pelo teste de Duncan a 5\%, após serem transformados em $\log (\mathrm{x}+1)$.

\section{RESULTADOS E DISCUSSÃO}

Todos os porta-enxertos e híbridos de pepino estudados apresentaram índice de galhas (IG) e de massa de ovos (IMO) igual a 5 , ou seja, apresentaram mais de 100 galhas e mais de 100 massas de ovos no sistema radicular, tanto para $M$. javanica (Tabela 1) como para $M$. incognita raça 2 (Tabela 2). O tomateiro 'Rutgers' proporcionou um FR igual a 21,8 e 17,8 para M. javanica e M. incognita raça 2, comprovando a viabilidade do inóculo utilizado.

Todos os porta-enxertos e híbridos de pepino estudados se comportaram como suscetíveis (ou hospedeiros) (Oostenbrink, 1966) a M. javanica (Tabela 1) e a $M$. incognita raça 2 (Tabela 2), ou seja, permitiram sua multiplicação. O fator de reprodução (FR) foi estatisticamente menor nos porta-enxertos do que nos híbridos de pepinos estudados para M. javanica (Tabela 1). Portanto, os híbridos de pepinos proporcionam maior 
Tabela 1. Peso da massa fresca de raiz (PFR), índice de galhas (IG), índice de massa de ovos (IMO), número de nematóides por grama de raiz (NN/g raiz), número de nematóides total (NNT) e fator de reprodução (FR) de Meloidogyne javanica em diferentes híbridos de pepino e porta-enxertos (root weight (PFR), gall index (IG), egg mass index (IMO), nematode number per root gram (NN/g raiz), total nematode number (NNT) and reproduction factor (FR) of Meloidogyne javanica in different cucumber hybrids and rootstocks). Botucatu, UNESP, 2008 .

\begin{tabular}{|c|c|c|c|c|c|c|}
\hline & $\operatorname{PFR}(\mathrm{g})$ & IG & IMO & NN/g raiz & NNT & FR \\
\hline \multicolumn{7}{|l|}{ Pepinos } \\
\hline Kouki & 47,7 a & 5 & 5 & 3733,4 a & $191.228 \mathrm{a}$ & 38,2 a \\
\hline Tsuyataro & $34,6 \mathrm{ab}$ & 5 & 5 & 3981,0 a & $132.578 \mathrm{a}$ & 26,5 a \\
\hline Taisho KY & 43,6 a & 5 & 5 & $2589,0 \mathrm{ab}$ & $121.574 \mathrm{a}$ & 24,3 a \\
\hline Yoshinari & $48,0 \mathrm{a}$ & 5 & 5 & $2042,3 \mathrm{abc}$ & $106.598 \mathrm{a}$ & $21.3 \mathrm{a}$ \\
\hline \multicolumn{7}{|l|}{ Porta-enxertos } \\
\hline Tetsukabuto & $37,1 \mathrm{ab}$ & 5 & 5 & 965,2 bcd & $42.934 \mathrm{~b}$ & $8,6 \mathrm{~b}$ \\
\hline Moranga Exposição & 25,9 bc & 5 & 5 & $1484,4 \mathrm{abc}$ & $38.992 \mathrm{bc}$ & $7,8 \mathrm{bc}$ \\
\hline B8-A Tetsukabuto & $28,5 \mathrm{bc}$ & 5 & 5 & $894,7 \mathrm{~cd}$ & $32.166 \mathrm{bcd}$ & $6,4 \mathrm{bcd}$ \\
\hline Excite Ikki KY & $16,7 \mathrm{~d}$ & 5 & 5 & $1122,9 \mathrm{bc}$ & $22.028 \mathrm{bcd}$ & $4,4 \mathrm{bcd}$ \\
\hline Menina Brasileira & $36,0 \mathrm{ab}$ & 5 & 5 & $542,0 \mathrm{~d}$ & $20.438 d$ & $4,1 \mathrm{~d}$ \\
\hline Ab. Shelper & $20,4 \mathrm{~cd}$ & 5 & 5 & $759,9 \mathrm{~cd}$ & $20.200 \mathrm{~cd}$ & $4,0 \mathrm{~cd}$ \\
\hline $\mathrm{CV}(\%)$ & 7,6 & & & 9,8 & 4,6 & 20,3 \\
\hline
\end{tabular}

*médias seguidas da mesma letra na coluna não diferem entre si pelo Teste de Duncan a 5\% de probabilidade (means followed by the same letter at column did not differ from each other by Duncan test at $5 \%$ of probability).

Tabela 2. Peso da massa fresca de raiz (PFR), índice de galhas (IG), índice de massa de ovos (IMO), número de nematóides por grama de raiz (NN/g raiz), número de nematóides total (NNT) e fator de reprodução (FR) de Meloidogyne incognita raça 2 em diferentes híbridos de pepino e porta-enxertos (root weight (PFR), gall index (IG), egg mass index (IMO), nematode number per root gram (NN/g raiz), total nematode number (NNT) and reproduction factor (FR) of Meloidogyne incognita in different cucumber hybrids and rootstocks). Botucatu, UNESP, 2008.

\begin{tabular}{|c|c|c|c|c|c|c|}
\hline & PFraiz (g) & IG & IMO & NN/g (g) & NNT & FR \\
\hline Pep. Yoshinari & 76,8 a & 5 & 5 & 2110,7 a & $153.486 \mathrm{a}$ & 30,7 a \\
\hline Pep. Taisho KY & 62,4 abc & 5 & 5 & 1841,9 a & $114.222 \mathrm{ab}$ & $22,8 \mathrm{ab}$ \\
\hline Pep. Kouki & $71,3 \mathrm{ab}$ & 5 & 5 & 2159,3 a & $139.244 \mathrm{abc}$ & $27,8 \mathrm{ab}$ \\
\hline Ab. Tetsukabuto & 34,1 e & 5 & 5 & 2036,9 a & $66.092 \mathrm{~cd}$ & 13,2 abc \\
\hline Ab. B8-A Tetsukabuto & $34,8 \mathrm{de}$ & 5 & 5 & $1216,5 \mathrm{a}$ & $50.500 \mathrm{abcd}$ & $10,1 \mathrm{abc}$ \\
\hline Ab. Excite Ikki KY & $19,4 \mathrm{f}$ & 5 & 5 & 1783,1 a & $45.042 \mathrm{ab}$ & 9,0 abc \\
\hline Ab. Moranga Exposição & $35,3 \mathrm{de}$ & 5 & 5 & $1121,8 \mathrm{a}$ & $44.242 \mathrm{abcd}$ & $8,8 \mathrm{abc}$ \\
\hline Ab. Shelper & $16,8 \mathrm{f}$ & 5 & 5 & $1529,1 \mathrm{a}$ & $37.652 \mathrm{bcd}$ & $7,5 \mathrm{bc}$ \\
\hline Ab. Menina Brasileira & 46,5 cde & 5 & 5 & 579,6 a & $30.788 \mathrm{~cd}$ & $6,2 \mathrm{c}$ \\
\hline Pep. Tsuyataro & $50,3 \mathrm{bcd}$ & 5 & 5 & 515,4 a & $25.246 \mathrm{~d}$ & $5,0 \mathrm{c}$ \\
\hline $\mathrm{CV}(\%)$ & 7,3 & & & 13,9 & 7,4 & 31,9 \\
\hline
\end{tabular}

*médias seguidas da mesma letra na coluna não diferem entre si pelo Teste de Duncan a $5 \%$ de probabilidade (means followed by the same letter at column did not differ from each other by Duncan test at $5 \%$ of probability).

aumento da população de $M$. javanica em um mesmo período de tempo, do que as abóboras que são utilizadas como porta-enxertos.

Os híbridos de pepino não diferiram estatisticamente entre si, apresentando fatores de reprodução de $M$. javanica elevados, variando de 21 a 38 . Estes valores são inferiores aos obtidos por Charchar \& Aragão (2005) em estufa
(66 a 170) e semelhantes aos a campo $(3,0$ a 59,7$)$. Entretanto, entre os porta-enxertos houve diferença significativa na reprodução do nematóide, demonstrada em todos os parâmetros analisados, sendo a abóbora 'Shelper' a que propiciou menor FR de $M$. javanica $(\mathrm{FR}=4,0)$; embora não tenha diferido estatisticamente dos FRs obtidos em 'Menina Brasileira' ( $F R=4,1)$, 'Excite
Ikki' $(\mathrm{FR}=4,4)$ e 'B8-A Tetsukabuto' $(\mathrm{FR}=6,4)$.

Também foi observada a multiplicação do $M$ incognita em todos os portaenxertos e híbridos de pepino estudados (Tabela 2), assim como no experimento com M. javanica. O pepino 'Tsuyataro' foi o que proporcionou menor fator de reprodução $(\mathrm{FR}=5,0)$ para esta espécie, embora não diferindo estatisticamente 
de todos os porta-enxertos: 'Menina Brasileira' (FR=6,2), 'Shelper' (7,5), 'Excite Ikki' $(\mathrm{FR}=9,0)$, 'Tetsukabuto' $(\mathrm{FR}=13,2)$ e 'B8-A Tetsukabuto' (FR= 13,2). Os híbridos de pepino 'Yoshinari', 'Taisho' e 'Kouki' apresentaram os maiores valores de fator de reprodução, superiores a 22. Carneiro et al. (2000), verificando a taxa de multiplicação de $M$. javanica, $M$. incognita raça 3, $M$. arenaria raça 2 e $M$. hapla em diferentes hortaliças, consideraram as abóboras 'Caserta' e 'Branca de Virgínea' resistentes apenas a $M$. hapla, assim como os pepinos 'SMR58' e 'Marketer'. As cultivares de pepinos e abóboras ora estudados apresentaram os fatores de multiplicação mais elevados aos obtidos por Carneiro et al. (2000).

Em uma área infestada apenas com M. incognita, o pepino 'Tsuyataro' não terá benefícios com a enxertia visando o manejo deste patógeno. Porém, em algumas áreas a infestação do solo ocorre com as duas espécies (M. incognita e $M$. javanica) conjuntamente (Charchar \& Moita, 2001). Nesta situação, as plantas enxertadas apresentarão menor multiplicação de pelo menos $M$. javanica ou de ambas as espécies do nematóide se o híbrido de pepino for o 'Yoshinari', 'Taisho' ou 'Kouki'.

Considerando-se a ausência de cultivares comerciais resistentes a $M$. javanica e $M$. incognita raça 2 , a adoção de mudas de pepinos enxertadas em áreas infestadas com esses nematóides, além de interferir de maneira positiva na qualidade e produtividade da cultura (Goto et al., 2003), pode também proporcionar, dependendo do porta-enxerto escolhido, menor multiplicação dessa espécie do nematóide das galhas comparada ao plantio de pés-franco dos híbridos estudados (Tabela 1). Entretanto, devese ressaltar que, embora os fatores de reprodução dos nematóides estudados sejam menores nos porta-enxertos que nos pepinos pé-franco, estes não são nulos, podendo elevar a população dos mesmos se cultivados de forma sucessiva em uma mesma área.

\section{REFERÊNCIAS}

BONETTI JI; FERRAZ S. 1981. Modificações do método de Hussey \& Barker para extração de ovos de Meloidogyne exigua em raízes de cafeeiro. Fitopatologia Brasileira 6: 553

CAÑIZARES KAL. 1998. A cultura de pepino. In: GOTO R; TIVELLI SW (eds). Produção de hortaliças em ambiente protegido: condições subtropicais. São Paulo: Fundação Editora UNESP. p. 195-223.

CAÑIZARES KAL; GOTO R. 1998. Crescimento e produção de híbridos de pepino em função da enxertia. Horticultura Brasileira 16: 110-112.

CARDOSO AII. 2009. Enxertia em pepino. Campo \& Negócio 52: 70-71.

CARDOSO AII; WILCKEN SRS. 2008. Nematóides assustam produtores de tomate e pepino. Campo \& Negócio 34: 38-39.
CARNEIRO RMDG; RANDIG O; ALMEIDA MRA; CAMPOS AD. 2000. Resistance of vegetable crops to Meloidogyne spp.: Suggestion for a crop rotation system. Nematologia Brasileira 24: 49-54.

CHARCHAR JM; ARAGÃO FAS. 2005. Reprodução de Meloidogyne spp. em cultivares de tomate e pepino sob estufa plástica e campo. Nematologia Brasileira 29: 243-249.

CHARCHAR JM; MOITAAW. 2001. Resistência de genótipos de batata a Meloidogyne javanica. Pesquisa Agropecuária Brasileira 36: 535540.

COOLEN WA; D' HERDE CJ. 2003. A method for quantitative extraction of nematodes from plant tissue. State Nematology end Entomology Research Station, Ghent. 77p.

GOTO R; SANTOS HS; CAÑIZARES KAL. Enxertia em hortaliças. São Paulo: Editora UNESP. 85p.

HUANG CS; VIANA BF. 1980. Relação entre níveis de inóculo pré-plantio de Meloidogyne incognita com o desenvolvimento do pepino. Fitopatologia Brasileira 5: 401-402.

HUSSEY RS; BARKER KR. 1973. A comparison of methods colleting inocula of Meloidogyne spp. including a new technique. Plant Disease Reporter 57: 1025-1028.

KAWAIDE T. 1985. Utilization of rootstocks in cucurbits production in Japan. Japanese Agricultural Research Quaterly 18: 285-288.

ODA M. 1995. New grafting methods for fruit-bearing vegetables in Japan. Japanese Agricultural Research Quaterly 29: 187-194.

ROBINSON RW; DECKER-WALTERS DS. 1999. Cucurbits. Cambridge: CAB International. 226p.

TAYLOR AL; SASSER JN. 1978. Biology, identification and control of root-knot nematodes (Meloidogyne species). Raleigh, North Carolina State University. 111p. 